\title{
Ultrafast Spectroscopy Studies on Thickness Dependence of Acoustic Phonon Modes in Silver Nanoprisms
}

\author{
Pyng $\mathrm{Yu}^{\mathrm{a}}$ (於 平), Ying-Jen Shiu* ( 許瑛珍), \\ Yit-Tsong Chen ${ }^{\mathrm{a}, \mathrm{b} *}$ ( 陳逸聰) and Sheng-Hsien $\operatorname{Lin}^{\mathrm{b}}$ ( 林聖賢) \\ ${ }^{a}$ Institute of Atomic and Molecular Sciences, Academia Sinica, P.O. Box 23-166, \\ Taipei 106, Taiwan, R.O.C. \\ ${ }^{\mathrm{b}}$ Department of Chemistry, National Taiwan University, Taipei 106, Taiwan, R.O.C.
}

\begin{abstract}
Time-resolved transient absorption technique is used to investigate the thickness dependence of acoustic phonon modes of silver nanoprisms with two thicknesses, $7.8 \pm 1.2$ and $8.5 \pm 0.69 \mathrm{~nm}$, and a similar bisector length of $31.4 \sim 31.6 \mathrm{~nm}$. Coherent acoustic phonon signals are observed. A new acoustic phonon frequency within $7.81 \mathrm{~cm}^{-1} \sim 11.7 \mathrm{~cm}^{-1}$ is found and this phonon mode is associated with the thickness of the nanoprism. Another phonon frequency between $1.95 \mathrm{~cm}^{-1}$ and $1.71 \mathrm{~cm}^{-1}$ is also observed, and its origin can be associated with the bisector length of the nanoprism.
\end{abstract}

Keywords: Nanoprism; Thickness correlated phonon.

\section{INTRODUCTION}

Recently, optical responses of silver nanoprisms have been investigated for possible applications on biosensors and communications. ${ }^{1-3}$ Compared with conventional nanospheroids, ${ }^{4}$ the nanoprisms exhibit a large red-shift of surface plasmon resonance (SPR). Due to a large tip curvature on a nanoprism, an induced local electric field in surface enhanced Raman scattering (SERS) can have 3500 incidences of electromagnetic field. ${ }^{5-7}$ In addition, the lateral surface (110) structure of a nanoprism provides much shorter-range chemical effects in SERS than the normal surface (111) structure. ${ }^{7}$ Several researchers have reported that the increased thickness of nanoprism results in a spectra shift about ten times larger than the case in which the bisector length of the nanoprism is elongated..$^{8-10}$

Raman scattering is an excellent technique used to observe a precise phonon frequency. ${ }^{11}$ However, as far as we know, there have been no reports of the phonon frequencies on nanoprisms by Raman scattering. Two totally symmetric acoustic phonon modes, correlated with the bisector length and half-edge length of silver nanoprism, have been reported by time-resolved transient absorption measurements. ${ }^{12-14}$ Although nanoprisms have three dimensions - bisector, edge length, and thickness - a phonon mode associated with the thickness of nanoprisms has not been observed. ${ }^{15}$ In this study, we are interested in a phonon mode related to the thickness of nanoprisms. Because the dimension of the thickness is much smaller than the other two dimensions, the corresponding phonon mode frequencies should be higher than the other two dimensions. It is possible to observe such higher frequencies if one scans the time-resolved transient absorption profiles with a smaller interval of the delay time-step. In this paper, we report a new phonon mode that is correlated with the thickness of the nanoprism.

\section{EXPERIMENTAL SECTION}

\section{Synthesis and characterizations}

A wet chemical method developed by Mirkin and his colleague ${ }^{8}$ was applied to synthesize two nanoprisms with different thicknesses. To an aqueous solution of silver nitrite, $\mathrm{AgNO}_{3},(0.1 \mathrm{mM}, 100 \mathrm{~mL})$, trosodium citrate, $\mathrm{Na}_{3} \mathrm{C}_{6} \mathrm{H}_{5} \mathrm{O}_{7},(30 \mathrm{mM}, 6 \mathrm{~mL})$, poly(vinylpyrrolidone) (PVP $\left.M_{w} \sim 55,000 \mathrm{~g} / \mathrm{mol}, 0.7 \mathrm{mM}, 6 \mathrm{~mL}\right)$, and hydrogen peroxide, $\mathrm{H}_{2} \mathrm{O}_{2}$, (35 wt.\%, $\left.240 \mu \mathrm{L}\right)$ were added in sequence and vigorously stirred at $23{ }^{\circ} \mathrm{C}$. Finally, a $100 \mathrm{mM}$ sodium borohydride, $\mathrm{NaBH}_{4}$, was injected into the mixture in the amounts of $500 \mu \mathrm{L}$ or $800 \mu \mathrm{L}$ in order to control the synthesized thickness of the nanoprisms. For about $20 \sim 40$ minutes, silver triangle nanoprisms were synthesized with the

\footnotetext{
* Corresponding author. E-mail: yingjen@gate.sinica.edu.tw
} 
indication that the yellow mixtures became blue. The concentration of the suspended silver triangle nanoprisms was estimated to be about $0.31 \mathrm{nM}$ in water solution after centrifugation (9000 prm, $8 \mathrm{~min}$ ).

The images of the synthesized nanoprisms were observed by a transmission electron microscope (TEM, Hitachi H-7100) operated at $75 \mathrm{kV}$, and the dimensional distribution of the silver nanoprisms were determined by dynamic light scattering (DLS, MALVERN, ZETASIZER300) method. All uv-vis absorption spectra of the silver nanoprisms solutions were measured by a spectrophotometer (JASCO V-570) with light path $10 \mathrm{~mm}$ of a cell.

\section{Time-resolved transient absorption measurements}

For the transient absorption measurements, the single color pump-probe technique was employed with the detailed experimental setup given elsewhere. ${ }^{16}$ A 100 fs visible-tunable laser provided by an optical parametric amplifier (OPA 9400, Coherent, Inc.) with a repetition rate of 20 $\mathrm{kHz}$ was applied to the nanoprisms for single color pumpprobe measurements. Here, the OPA laser was generated by an amplifier of a Ti:sapphire laser system (RegA 9000, Coherent, Inc.), in which a RegA laser was pumped and seeded by an argon ion laser (Innova 400, Coherent, Inc.) and an oscillator of the Ti:sapphire laser system (Mira 900, Coherent, Inc.). A translational stage was performed to control delay time between the pump and probe beams, which were focused on the samples with a laser energy ratio of 10 to 1 . The probed beam was detected by a photodiode detector (2001-FC, New Focus Inc.) and acquired by a personal computer through a lock-in amplifier (SR830, Stanford Research Inc.). A perpendicular polarization between the two beams was employed to filter out the scattered light of the pump beam which was chopped with a frequency of $1 \mathrm{kHz}$. To avoid the conformation change, a low pump power density of $\sim 1.0 \times 10^{3} \mathrm{~W} / \mathrm{cm}^{2}$ was employed in each sample system. Here, the samples were stored in an ice bath and a dark environment and circulated in a flow cell in a $1 \mathrm{~mm}$ optical light path to reduce the photo-degradation during the measurements.

\section{RESULTS AND DISCUSSION}

To obtain the phonon frequency associated with the thickness of the nanoprism, we first characterized the di- mensions of the two synthesized nanoprisms by TEM images. One is a thicker nanoprism with a thickness of $D=8.5$ $\pm 0.7 \mathrm{~nm}$ and a bisector length of $H=31.4 \pm 8.5 \mathrm{~nm}$; the other one is thinner with $D=7.8 \pm 1.2 \mathrm{~nm}$ and $H=31.6 \pm$ $7.1 \mathrm{~nm}$. Photo images, including the top and side views of the synthesized nanoprisms, are shown in panels (a1)-(a3) in Fig. 1, and the bisectors distribution are presented in panels (b1) and (b2), also in Fig. 1. The dimensional distribution of the two nanoprisms is also measured by DLS, in which, as shown in Fig. 1(c), about $20 \%$ and $10 \%$ residues (dimension $<5 \mathrm{~nm}$ ) exist for nanoprisms $D=8.5 \mathrm{~nm}$ and $D$ $=7.8 \mathrm{~nm}$, respectively. In addition, the UV-visible absorption measurements are also performed to characterize the

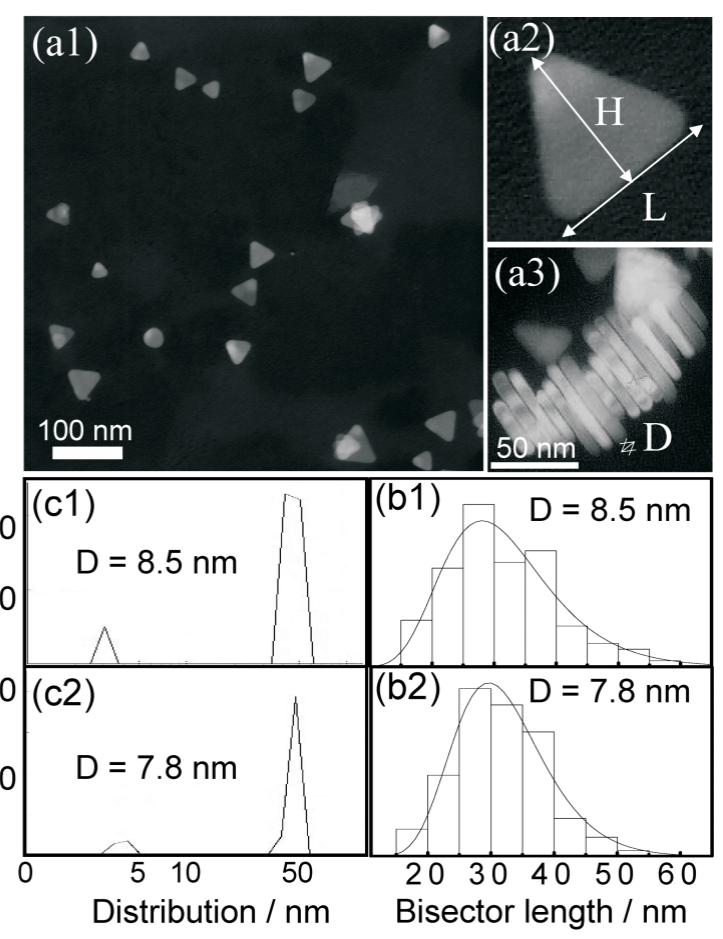

Fig. 1. (a) The quality of synthesized silver nanoprisms is illustrated by TEM image. The nanoprisms are deposited on a carbon film coated on a copper grid. A top view of a single nanoprism is exhibited, where $H$ and $L$ indicate the bisector length and the edge length, respectively. Stacks of silver nanoprisms assembled in a top-to-base manner on the carbon film are also presented, in which $D$ indicates the thickness. (b) The histograms of statistical dimensions of the bisectors length of the nanoprism $D=8.5$ nm and $D=7.8 \mathrm{~nm}$ are about $100-200$ particles. (c) The DLS results display the size distribution of nanoprisms for $D=8.5 \mathrm{~nm}$ and $D=7.8 \mathrm{~nm}$. 
synthesized silver nanoprisms. A blue shift of the SPR band from $680 \mathrm{~nm}$ to $621 \mathrm{~nm}$ is observed by increasing the thickness from $D=7.8 \mathrm{~nm}$ to $D=8.5 \mathrm{~nm}$, as shown in Fig. 2 . Since the blue shift of the in-plane dipole SPR transition depends on three factors, namely, the edge length, the snipped tips, and the thickness of nanoprisms, ${ }^{4,8,9}$ the shift is due to the thickness of the nanoprisms for the case of a similar edge length and comparable snipped tips. It is noted that less absorption at $400 \mathrm{~nm}$ suggests a negligible concentration of residues and negligible spherical nanoparti-

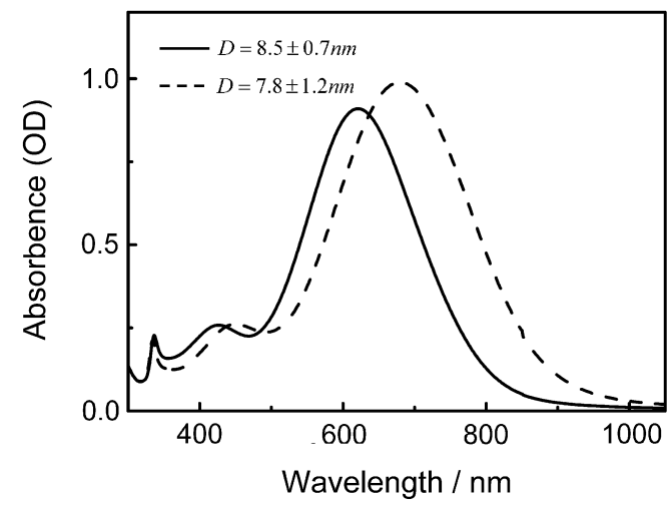

Fig. 2. The UV-visible absorption spectra of both silver nanoprisms. The solid line is for the nanoprism $D=8.5 \mathrm{~nm}$ and the dashed line is for the one where $D=7.8 \mathrm{~nm}$. cles (NPs) in the solution.

To investigate thickness dependence of acoustic phonons of nanoprisms, the single color pump-probe technique is employed in which the nanoprism is excited upon the SPR absorption band with excitation wavelengths at 575 $\mathrm{nm}$ and $640 \mathrm{~nm}$ for $D=8.5 \mathrm{~nm}$ and $D=7.8 \mathrm{~nm}$, respectively. By adjusting the interval of the time steps in the pump-probe experiments, two types of coherent phonons are observed from each of the two different thickness nanoprisms. Panels (a1) and (a2) in Figs. 3 and 4 show the observed time-resolved profiles within a delay time of 100 psec and that of $8.5 \mathrm{psec}$, correspondingly. The scattered points represent the observed transient absorption data and the solid lines denote the decay components. After subtracting the decay components from the observed time-resolved profiles, the oscillation components can be deduced as shown in panels (b1) and (b2) in Figs. 3 and 4. The Fast Fourier Transform (FFT) can be employed to deduce the phonon frequencies, which are also examined by the single value deconvolution (SVD) method ${ }^{16}$ as shown in panels (c1) and (c2) in Figs. 3 and 4. The consistent spectra analyzed by FFT and SVD assure that low and high acoustic phonon frequencies, $v_{\ell}$ and $v_{h}$, are obtained. Two frequencies of $1.95 \pm 0.1 \mathrm{~cm}^{-1}$ and $7.81 \pm 4.0 \mathrm{~cm}^{-1}$ are obtained for nanoprism $D=8.5 \mathrm{~nm}$, and those of $1.71 \pm 0.1 \mathrm{~cm}^{-1}$ and

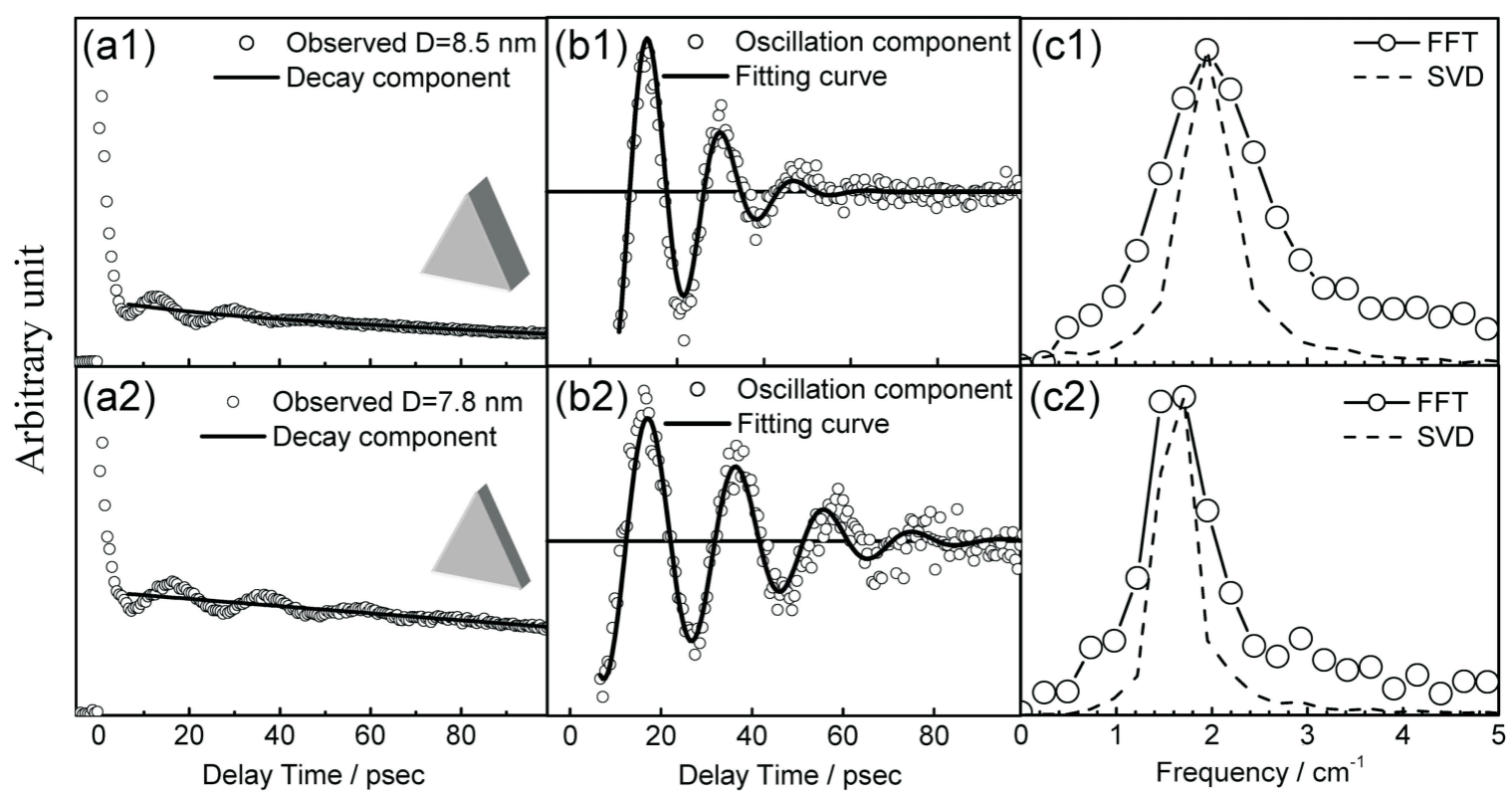

Fig. 3. (a) The observed transient absorption profiles (circles) within $100 \mathrm{psec}$ of delay time and the deduced decay components (solid lines). (b) The deduced oscillation components (circles) and fitting curve (solid lines). (c) The FFT (circles and lines) and SVD (dashed line) analysis spectra of the low frequency phonon mode. 


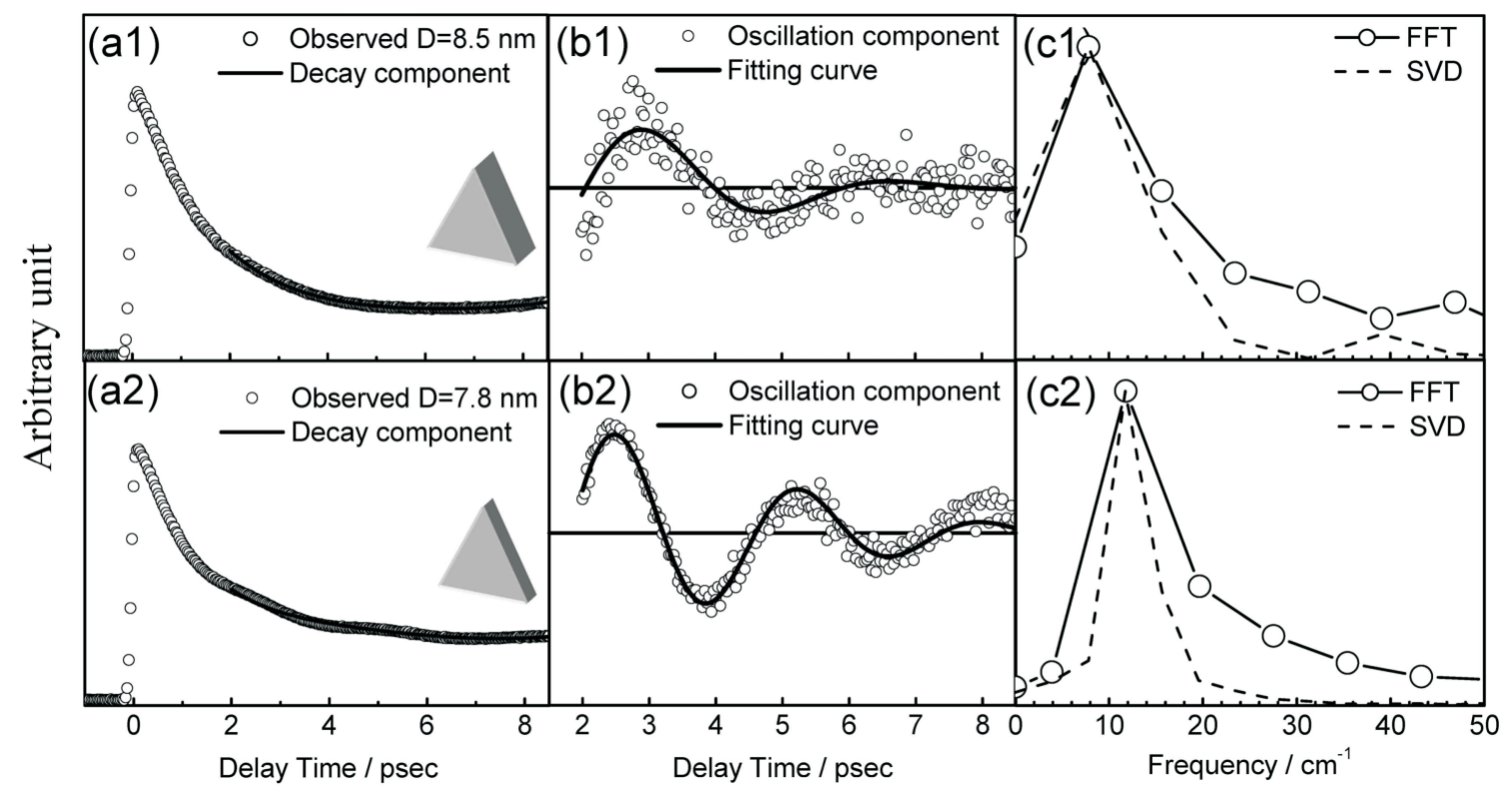

Fig. 4. (a) The observed transient absorption results (circles) within $8.5 \mathrm{psec}$ of delay time and deduced decay components (solid line). (b) The deduced oscillation component (circles) and fitting curve (solid line). (c) FFT (circles and lines) and SVD (dashed line) analysis spectra of the high frequency phonon mode.

$11.7 \pm 4.0 \mathrm{~cm}^{-1}$ are for $D=7.8 \mathrm{~nm}$. Their correspondence phonon time constants $T$ are $17.1 \mathrm{psec}$ and $4.27 \mathrm{psec}$ for the nanoprism with $D=8.5 \mathrm{~nm}$ and $19.5 \mathrm{psec}$ and $2.85 \mathrm{psec}$ for the one with $D=7.8 \mathrm{~nm}$. Here, the uncertainty of the deduced phonon frequency $\Delta$ is determined by the number of the data points $N$ and the step size of the delay time $\Delta t$ such that $\Delta=1 /(c \times \Delta t \times N)$ where $c$ is the velocity of light.

Furthermore, a phenomenological approximation is adopted to identify the deduced frequencies of nanoprisms because the derivation of the phonon frequencies of a nanoprism based on the continuum elastic theory is not available. On top of that, numerical simulation of the system is extremely demanding. With $v=c_{L} / 2 c H,{ }^{12}$ the frequency of $1.9 \mathrm{~cm}^{-1}$ is obtained as $H=31.4 \mathrm{~nm}(D=8.5 \mathrm{~nm})$ and $H=$ $31.6 \mathrm{~nm}(D=7.8 \mathrm{~nm})$, where $c_{L}=3650 \mathrm{~m} / \mathrm{s}$ is the longitudinal sound speed in the bulk of silver. The consistency between the calculated frequency and the observed low frequency indicates that the acoustic phonon frequency $v_{\ell}$ can be associated with the bisector length. This phonon mode, in fact, has been assigned to be a symmetric breathing mode for the nanoprism at edge length $50-120 \mathrm{~nm} .^{13,14}$ Moreover, a thickness-correlated phonon frequency has been reported for polycrystalline silver film with thickness $23 \mathrm{~nm}$ based on the equation $v=c_{L} / 2 c D \cdot{ }^{17,18}$ It is practical at this moment for us to adopt the same equation to interpret the phonon frequency associated with the thickness of the nanoprism. The calculated frequencies are given as 7.2 $\mathrm{cm}^{-1}$ for $D=8.5 \mathrm{~nm}$ and $7.8 \mathrm{~cm}^{-1}$ for $D=7.8 \mathrm{~nm}$, respectively. These values are found to be slightly smaller than the observed high frequencies $v_{h}$; nevertheless, the estimated phonon frequencies are within the uncertainty of the observed one $v_{h}, 7.81 \pm 4.0 \mathrm{~cm}^{-1}$ and $11.7 \pm 4.0 \mathrm{~cm}^{-1}$. Thus, we propose that the high phonon frequency mode can be associated with the thickness of the nanoprisms. All the observed and calculated phonon frequencies are summarized and listed in Table 1. It is noted that the phenomenological approximation ignores the correlation effect between the dimensions of the bisector length and the thickness. In addition, the observed phonon frequencies contributed from the negligible amount of residues and the spherical NPs should be ignored at the applied excitation wavelengths. The totally symmetric phonon mode associated with the half-edge length of a nanoprism is not observed in this work ${ }^{14}$ probably due to the very weak amplitude of the phonon mode.

The time-development of a coherent acoustic phonon oscillation can be expressed by

$$
S(t)=A \cos \left(\frac{2 \pi t}{T}+\phi\right) \exp \left(-(t / \tau)^{2}\right),
$$


Table 1. The observed and calculated acoustic phonon frequencies of both silver nanoprisms

\begin{tabular}{lcccc}
\hline & \multicolumn{2}{c}{$D=8.5 \mathrm{~nm}$} & \multicolumn{2}{c}{$D=7.8 \mathrm{~nm}$} \\
\cline { 2 - 5 } & Observed (SVD) & $\begin{array}{c}\text { Phenomenological } \\
\text { analysis }\end{array}$ & Observed (SVD) & $\begin{array}{c}\text { Phenomenological } \\
\text { analysis }\end{array}$ \\
\hline$v_{\ell}\left(\mathrm{cm}^{-1}\right)$ & $1.95 \pm 0.1$ & 1.9 & $1.71 \pm 0.1$ & 1.9 \\
$v_{h}\left(\mathrm{~cm}^{-1}\right)$ & $7.81 \pm 4.0$ & 7.2 & $11.7 \pm 4.0$ & 7.8 \\
\hline
\end{tabular}

Table 2. The amplitude ratios, damping time constants, and phases of two phonon modes of both nanoprisms

\begin{tabular}{lcc}
\hline $\begin{array}{l}\text { Thickness correlated mode/ } \\
\text { Bisector correlated mode }\end{array}$ & $D=8.5 \mathrm{~nm}$ & $D=7.8 \mathrm{~nm}$ \\
\hline Amplitude ratio & $0.192 / 1$ & $0.778 / 1$ \\
$\tau(\mathrm{psec}) / \tau(\mathrm{psec})$ & $4.11 / 27.8$ & $5.10 / 45.6$ \\
$\phi(\mathrm{deg}) / \phi(\mathrm{deg})$ & $78.5^{\circ} / 79.1^{\circ}$ & $44.3^{\circ} / 42.4^{\circ}$ \\
\hline
\end{tabular}

where $A, \phi$, and $\tau$ are the amplitude, phase, and damping time constant, respectively. For the thickness-correlated phonon mode, with application of $T=4.27 \mathrm{psec}$ and $T=$ 2.85 psec to Eq. (1) for the nanoprism $D=8.5 \mathrm{~nm}$ and $D=$ $7.8 \mathrm{~nm}, \phi$ are obtained as $78.5^{\circ}$ and $44.3^{\circ}$, and $\tau$ are 4.11 psec and 5.10 psec, respectively. By using $T=17.1 \mathrm{psec}$ and $T=19.5 \mathrm{psec}$ for the bisector-correlated phonon mode of the corresponding nanoprisms, $\phi$ are determined as $79.1^{\circ}$ and $42.4^{\circ}$, and $\tau$ are $27.8 \mathrm{psec}$ and $45.6 \mathrm{psec}$, respectively. The amplitude ratio of the thickness-correlated mode to the bisector-correlated mode is $0.192 / 1(0.778 / 1)$ for the nanoprism $(D=7.8 \mathrm{~nm})$. All deduced results are listed in Table 2 and the simulated profiles are displayed in panels (b1) and (b2) in Figs. 3 and 4. The smaller damping time constant for nanoprism $D=8.5 \mathrm{~nm}$ is due to the larger dimension fluctuation as shown in the DLS results (see panels (c1) and (c2) in Fig. 1). Similarity in the phases of both phonon modes observed from one nanoprism solution confirm that the deduced phonon frequencies $v_{\ell}$ and $v_{h}$ can be originated from the same nanoprisms, while the phonon phase can be different at various probed wavelengths. ${ }^{14}$ The phonon phase of the thickness-correlated mode and a mechanism of electron-phonon coupling are still under investigation.

\section{CONCLUSIONS}

New acoustic phonon frequencies within $7.81 \mathrm{~cm}^{-1}$ and $11.7 \mathrm{~cm}^{-1}$ are found in the femto-second time-resolved transient absorption profiles of silver nanoprisms with thicknesses of $8.5 \pm 0.69 \mathrm{~nm}$ and $7.8 \pm 1.2 \mathrm{~nm}$ after analysis by FFT and SVD methods. The newly observed frequencies are suggested to be a thickness-correlated phonon mode. The bisector-correlated phonon frequency of 1.95 $\mathrm{cm}^{-1}\left(1.71 \mathrm{~cm}^{-1}\right)$ is also observed for the nanoprism with a bisector of $31.4 \pm 8.5 \mathrm{~nm}(31.6 \pm 7.1 \mathrm{~nm})$. The amplitudes ratio, phases, and damping time constants of both phonon modes are also deduced from an analysis of the time-development behaviors of the observed coherent acoustic phonon.

\section{ACKNOWLEDGEMENTS}

We thank Prof. H.-T. Chang and Dr. Y.-F. Huang for their helpful suggestions on the sample preparations. We acknowledge the Instrumental Center of National Taiwan University for TEM conduction. This work is supported by the National Science Council of the ROC and Academia Sinica.

Received June 12, 2007.

\section{REFERENCES}

1. Jain, P. K.; Lee, K. S.; El-Sayed, I. H.; El-Sayed, M. S. J. Phys. Chem. B 2006, 110, 7238.

2. Haes, A. J.; Zou, S.; Schatz, G. C.; Van Duyne, R. P. J. Phys. Chem. B 2004, 108, 109.

3. Bastys, V.; Pastoriza-Santos, I.; Rodríguez-González, B.; Vaisnoras, R.; Liz-Marzán, L. M. Adv. Funct. Mater. 2006, $16,766$.

4. Kelly, K. L.; Coronado, E.; Zhao, L. L.; Schatz, G. C. J. Phys. Chem. B 2003, 107, 668.

5. Hao, E.; Schatz, G. C.; Hupp, J. T. J. Fluoresce. 2004, 14, 331.

6. Singh, A.; Chaudhari, M.; Sastry, M. Nanotechology 2006, $17,2399$.

7. Zhang, J.; Li, X.; Sun, X.; Li, Y. J. Phys. Chem. B 2005, 109, 12544.

8. Metraux, G. S.; Mirkin, C. A. Adv. Mater. 2005, 17, 412. 
9. Jin, R.; Cao, Y. C.; Hao, E.; Métraux, G. S.; Schatz, G. C.; Mirkin, C. A. Nature 2003, 425, 487.

10. Hao, E.; Schatz, G. C.; Hupp, J. T. J. Fluoresce. 2004, 14, 331.

11. Portales, H.; Saviot, L.; Duval, E.; Fujii, M.; Hayashi, S.; Del Fatti, N.; Vallée, F. J. Chem. Phys. 2001, 115, 3444.

12. Huang, W.; Qian, W.; El-Sayed, M. A. Nano Lett. 2004, 4, 1741.

13. Hu, M.; Petrova, H.; Wang, X.; Hartland, G. V. J. Phys. Chem. B 2005, 109, 14426.

14. Bonacina, L.; Callegari, A.; Bonati, C.; van Mourik, F.;
Chergui, M. Nano Lett. 2006, 6, 7.

15. Huang, W.; Qian, W.; El-Sayed, M. A. J. Phys. Chem. B 2005, 109, 18881.

16. Zhang, J. M.; Shiu, Y. J.; Hayashi, M.; Liang, K. K.; Chang, C. H.; Gulbinas, V.; Yang, C. M.; Yang, T.-S.; Wang, H. Z.; Chen, Y.-T.; Lin, S. H. J. Phys. Chem. A 2001, 105, 8878.

17. Fatti, N. D.; Voisin, C.; Christofilos, D.; Vallée, F.; Flytzanis, C. J. Phys. Chem. A 2000, 104, 4321.

18. Lai, W. M.; Rubin, D.; Krempl, E. Introduction to Continuum Mechanics; Pergamon: New York, 1993; pp 251-253. 